\title{
Prescription appropriateness of empiric antibiotic therapy: survey in the internal medicine departments of the Emilia Romagna region
}

\author{
Giovanni Pedretti, ${ }^{1}$ Pasquale Gianluca Giuri, ${ }^{2}$ Giuseppe Chesi, ${ }^{3}$ Giuseppe Civardi, ${ }^{4}$ Giovanni Dall'Asta, ${ }^{5}$ \\ Matteo Giorgi Pierfranceschi, ${ }^{6}$ Giorgio Cioni ${ }^{7}$ for the Steering Committee FADOI Emilia Romagna \\ ${ }^{1}$ Internal Medicine Department, Vaio Hospital, Fidenza (PR); ${ }^{2}$ Internal Medicine Department, S. Anna Hospital, Castelnovo \\ né Monti (RE); ${ }^{3}$ Internal Medicine Department, Magati Hospital, Scandiano (RE); ${ }^{4}$ Internal Medicine Department, Hospital \\ of Val d'Arda, Fiorenzuola d'Arda (PC); ${ }^{5}$ Italian Association of Epidemiology (AIE), Roma; ${ }^{6}$ Emergency Department, \\ Hospital of Val d'Arda, Fiorenzuola d'Arda (PC); ${ }^{7}$ Internal Medicine Department, Pavullo nel Frignano Hospital (MO), Italy
}

\begin{abstract}
The article reports the results of an observational study conducted in 53 internal medicine departments of the Emilia Romagna region based on the answers to 54 questions administered in a questionnaire, submitted electronically, based on the type of center (large $>250$ beds, small $<250$ beds), laboratory equipment, management aspects, pharmacological and clinical/economic aspects and use of antibiotics in the empirical treatment of severe infections. The result is a snapshot of the existing situation which shows a substantial availability of resources and a good level of expertise in the field of infectious disease management, despite some areas still need improvement. It also highlights some differences in terms of procedures amongst large hospitals, where infectious diseases are treated by an ad hoc specialized staff, and the small ones, where the internist is generally involved, being responsible for both direct management of severe infections and giving advice to other departments (emergency or surgery). It emerged that, both in small and large hospitals, more discussion and continuous assessment are needed to apply the appropriate protocols based on reference checklists.
\end{abstract}

\section{Introduction}

The internist is often called to the front line, especially in peripheral hospitals, to address the problem of empirical antibiotic therapy to treat severe infections requiring hospitalization, because not all hospitals can rely on professionals with a specific knowledge of infectious diseases. For this reason the first diagnosis of

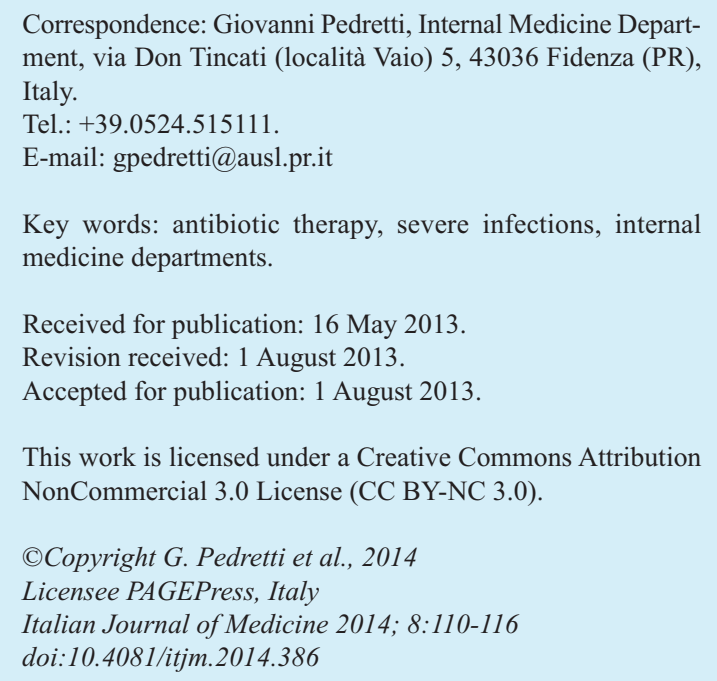

these cases and the correct implementation of treatment fall within the responsibility of the internist and are extremely important steps to ensure the survival of patients and an appropriate use of resources. It is well known that the outcome, especially in severe infections, is strongly influenced by the choice of the right antibiotic, the timing of the initial administration, its appropriate dose and its correct use. ${ }^{1-4}$

Having pharmaco-economic constraints and sometimes unsatisfactory tools to assess the appropriateness of prescription, internists are encouraged to take on an active role in the formulation of tools and actions targeted to therapeutic appropriateness and its continuous assessment through specific indicators. Some preliminary activities in this field provided favorable indications about the clinicians' compliance to prescription guidelines prepared by expert panels and their favorable impact in terms of resource rationalization..$^{5-7}$ Some surveys show that prescription appropriateness, built on shared guidelines and measured with appropriate tools compatible with the clinical activity, can boost efficacy and effectiveness, which are the two main objectives of clinical management, in spite of differences among individual hospitals. ${ }^{8-10}$

The purpose of our study is to enhance therapeutic appropriateness and its continuous assessment in all internal medicine departments in the Emilia Romagna region. 


\section{Materials and Methods}

The experimental design of the study includes several phases, as summarized below.

- Administration of a questionnaire to all the internal medicine teams in the region in order to have a snapshot of the existing situation in terms of: infectious disease management, use and availability of diagnostic tools (microbiology laboratories, specific blood tests, etc.), patterns of antibiotics use for empiric treatment of the most frequent infections managed by the internists (sepsis, pneumonia, urinary tract infections, soft tissue infections, endocarditis, abdominal infections etc.); use of reporting systems on the use of antibiotics.

- Clinical and statistical analysis of the data and epicrisis with proposals for improvement.

- Feedback with acquired data and recommended corrective actions in an ad-hoc publication addressed to all internal medicine departments in the region.

- Assessment of the implementation of measures for improvement and the consistency of clinical behavior according to agreed indicators (self-assessment checklist to be used during internal clinical audit).

The questionnaire, prepared on a word form, was sent as an attachment to an e-mail to the 80 internal medicine departments in the region, in order to take a snapshot of the situation at time 0 . The form included 54 closed questions with a yes/no answer on 5 datasets: hospital, laboratory, management of patients with infection, use of antibiotics and organizational features. For each Department a single operator, either the head or his/her delegate, was requested to answer.

The groups of questions were focused on the following topics:

- Eight questions were on the structure (hospital and departments): total number of beds, number of beds in the department, presence at the hospital of reference units responsible for managing critical illnesses in general and severe infections in particular [emergency room (ER), intensive care unit (ICU), infectious disease department or isolation unit, organization by care intensity];

- Six questions were on the reference clinical analysis laboratory (availability for emergency service $24 \mathrm{~h}$ a day, availability of microbiology laboratory, availability of mimimum inhibitory concentrations (MICs) for various antibiotics and some serological tests for severe infections: procalcitonin, and serum antibiotics assays);

- Eleven questions were on management to estimate the attention paid by the internal medicine department and, more generally, the hospital to the problem of severe infections and their treatment: availability of a committee in charge of hospital infections, possibility of placing a central venous catheter (CVC) within $24 \mathrm{~h}$ from the onset of severe sepsis, protocols for sampling and prophylaxis in surgery, availability of professional experience in the field of antibiotic therapy and advice from the internal medicine department for severe infections in the ER and surgical wards;

- Twenty-one questions were on pharmacologic aspects in order to investigate how appropriately the different classes of antibiotics are used for some infectious diseases, since this has implications in particular on the incidence, the severity and the impact on the organization in terms of clinical work and use of resources;

- Eight questions were on the clinical-economic organization in relation to the use of antibiotics, prescription appropriateness, economic reporting and any innovative ideas on management.

\section{Statistical analysis}

The feedback data was entered into a single Microsoft Excel 2007 spreadsheet and then commented in a report by simply counting the positive and negative answers on the entire population of departments that filled in the questionnaire. The second part of the analysis involves a binary stratification of data into two distinct groups based on the size of the hospital, defined according to the number of beds (distinguishing between large structures or hubs with over 250 beds and small structures or spokes with less than 250 beds). Then the presence of statistical associations between answers to some questions and the size of the hospital was assessed between these two groups, using the $\chi$ test and considering statistically significant data with P-values $<0.05$.

\section{Results}

Fifty-three departments $(66.25 \%)$ out of the 80 contacted filled in the questionnaire with a good distribution among the various centers in the Region (Figure 1).

University and hospital departments participated. The characteristics of the various structures are shown in Figure 2.

In almost all structures there is an ER (98\%) and a long-term care department for post-acute conditions (86.5\%).

As to the reference laboratories, $77.4 \%$ of the internal medicine departments have a facility for microbiological tests at the hospital with a 24/7 emergency service and qualified to test antibiotics' MIC sensitivity in $93.5 \%$ of the cases. It is also possible to have procalcitonin serum assays in $73 \%$ of the cases and antibiotics serum assays (gentamicin, vancomycin) in $80.4 \%$ of the cases. 
As to the management of patients with severe infectious diseases, only in $30.2 \%$ of the cases the internal medicine departments can place a CVC independently with no difference between large and small hospitals ( $32 \%$ vs $32.1 \%$, n.s.); but $94.7 \%$ of the departments unable to do so can rely on a coded collaboration scheme with other departments (surgery, ICU) that can place a CVC within $24 \mathrm{~h}$. In $92.5 \%$ of the cases there is a Committee for hospital infections both in large and small hospitals $(100 \% v s$ $84 \%$ n.s.) and in $92.2 \%$ of the cases there is a protocol for infection prophylaxis in surgery. In slightly over half of the cases (50.9\%) there is a physician trained in antibiotic therapy with no difference between large and small hospitals (53.6\% vs $48 \%$ n.s.). By contrast, in the case of patients with severe infections, the ER contacts the internal medicine department for advice in $45.3 \%$ of the cases with a statistically significant difference $(32 \%$ vs $62 \%, \mathrm{P}<0.05)$ between small hospitals and large hospitals, where such advice is more often requested to the infectious disease department. Likewise in $75 \%$ of the cases the surgical wards ask for advice to internists for patients with severe infections. Once again, this occurs more frequently in small hospitals than in large ones (57\% vs $92 \%$ $\mathrm{P}<0.05)$. How blood cultures are managed according to shared protocols is shown in Figure 3. As can be seen, a great deal of attention is paid to the initial blood culture at the beginning of any antibiotic ther- apy in order to reach a correct microbiological diagnosis and select the most appropriate treatment.

As to the selection of drugs, only half of the departments $(50.9 \%)$ have an official manual on the use of antibiotics with no difference between the two types of structures; aminoglycosides are used in a daily singledose regimen in almost all cases (92.5\%); in most cases quinolone is not used as first choice in urinary tract infections (66\%), while a 3-5 h infusion of carbapenems is administered only in 59.6\%; glycopeptides are still used as first choice in a high percentage $(51.9 \%)$ of methicillin-sensitive Staphylococcus aureus infections (MSSA); the antibiotic loading dose is not given in $58.5 \%$ of the cases where it is required, with no difference between large and small hospitals. A statistically significant difference can be identified in the use of innovative drugs, especially in patients with methicillinresistant Staphylococcus aureus infections (MRSA) with high MIC for glycopeptides: tigecycline in 41.5\% of the cases ( $50 \%$ vs $32 \%$, n.s.), linezolid in $68 \%$ of the cases $(78.6 \%$ vs $56 \%, \mathrm{P}<0.05)$, daptomycin in $26 \%$ of the cases $(32.1 \%$ vs $20 \%, \mathrm{P}<0.05)$.

As to the data on the organization, reports on the antibiotic therapy are available only in $47.1 \%$ of the cases with a significantly higher score in large hospitals than in smaller ones $(60.7 \%$ vs $28 \% \mathrm{P}<0.05)$. Unfortunately these reports focus exclusively on economic aspects $(61.5 \%)$ rather than prescription appropriateness. However, they are computerized

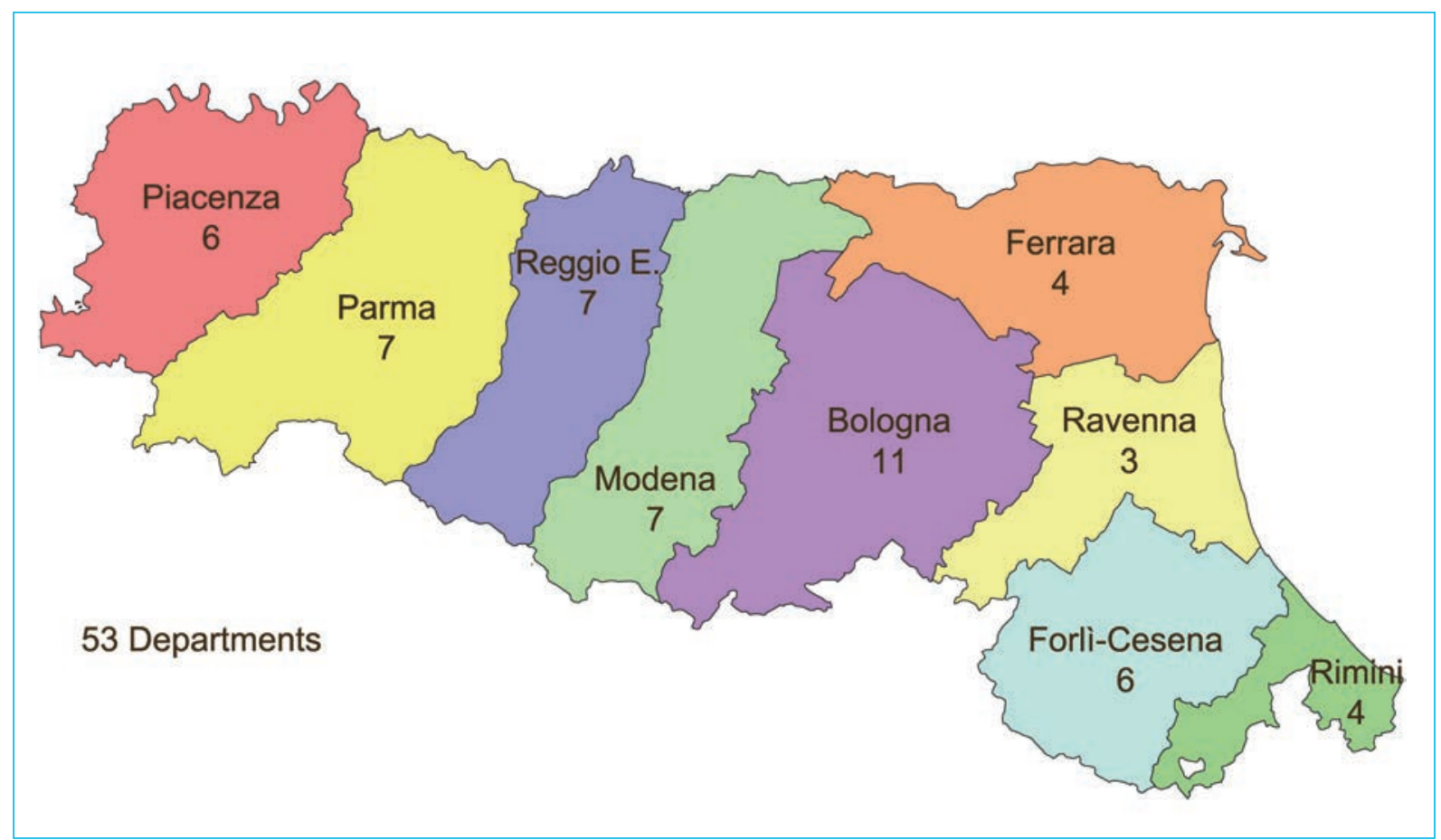

Figure 1. Geographical distribution of internal medicine departments which participated in the survey. 
(67.3\%) mainly in small hospitals (76\%) compared with large ones $(50 \%, \mathrm{P}<0.05)$. The feedback on management data is given systematically to the clinicians (72.5\%), but, systems for measuring the results are not frequently tested $(60.8 \%)$, although this occurs significantly more often in smaller hospitals than in larger ones ( $48 \%$ vs $28.6 \%, \mathrm{P}<0.05)$. The use of computerized drug prescriptions is not yet common in the region $(26 \%)$ and can be found significantly more often in large hospitals (39.3 vs $24 \%, \mathrm{P}<0.05)$.

Table 1 includes a summary of the statistically significant variables.

\section{Discussion and Conclusions}

One of the latest and most exciting chapters of internal medicine today is the antibiotic therapy for severe infections that commonly occur in our hospitals.

Several authors have shown that an early antibiotic therapy with the right timing, based on an adequate choice of the molecule, driven by the antibacterial activity and by the local ecology of infectious diseases, administered with the right dose regimen and for a suitable period of time is key to ensure both clinical efficacy and long survival. Numerous studies have shown that in critically ill patients the full implementation of the empirical antibiotic therapy within $3 \mathrm{~h}$ from admission ensures a statistically significant drop in mortality from $56 \%$ to $33 \%(\mathrm{P}<0.03) .{ }^{9}$ They have also shown that this approach is just as effective as an adequate hydration and ventilation of patients with septic shock. However, it is not enough to know the characteristics of the antimicrobial spectrum of a molecule, it is also important to know and apply the principles of pharmacokinetics, pharmacodynamics and the kinetic/dynamic correlations occurring especially in the case of both Gram+ and Gram- multi-resistant bacteria against which the therapeutic armamentarium appears extremely limited. In these cases, applying the criteria based on absorption, distribution, metabolism and elimination of the drug is a priority to promote good penetration into the infection site and to obtain the required plasma and tissue concentrations to eradicate the germs. This cannot be separated from the knowledge of the chemical characteristics of the antibiotics (hydrophilic or lipophilic), their dynamic characteristics (time-dependent or dose-dependent) and of course the patient's condition. Patients with increased volume of drug distribution require a higher loading dose, whereas patients with increased or decreased elimination due to impairment of excretory organs (kidney and/or liver) require respectively higher or lower maintenance doses. Other factors that must be taken into account, when implementing the antibiotic therapy, are the availability of a protocol for the collection of tests on blood and other body fluids, that must be already in place before the start of empirical therapy and be formulated and shared with the whole medical and nursing staff and finally the

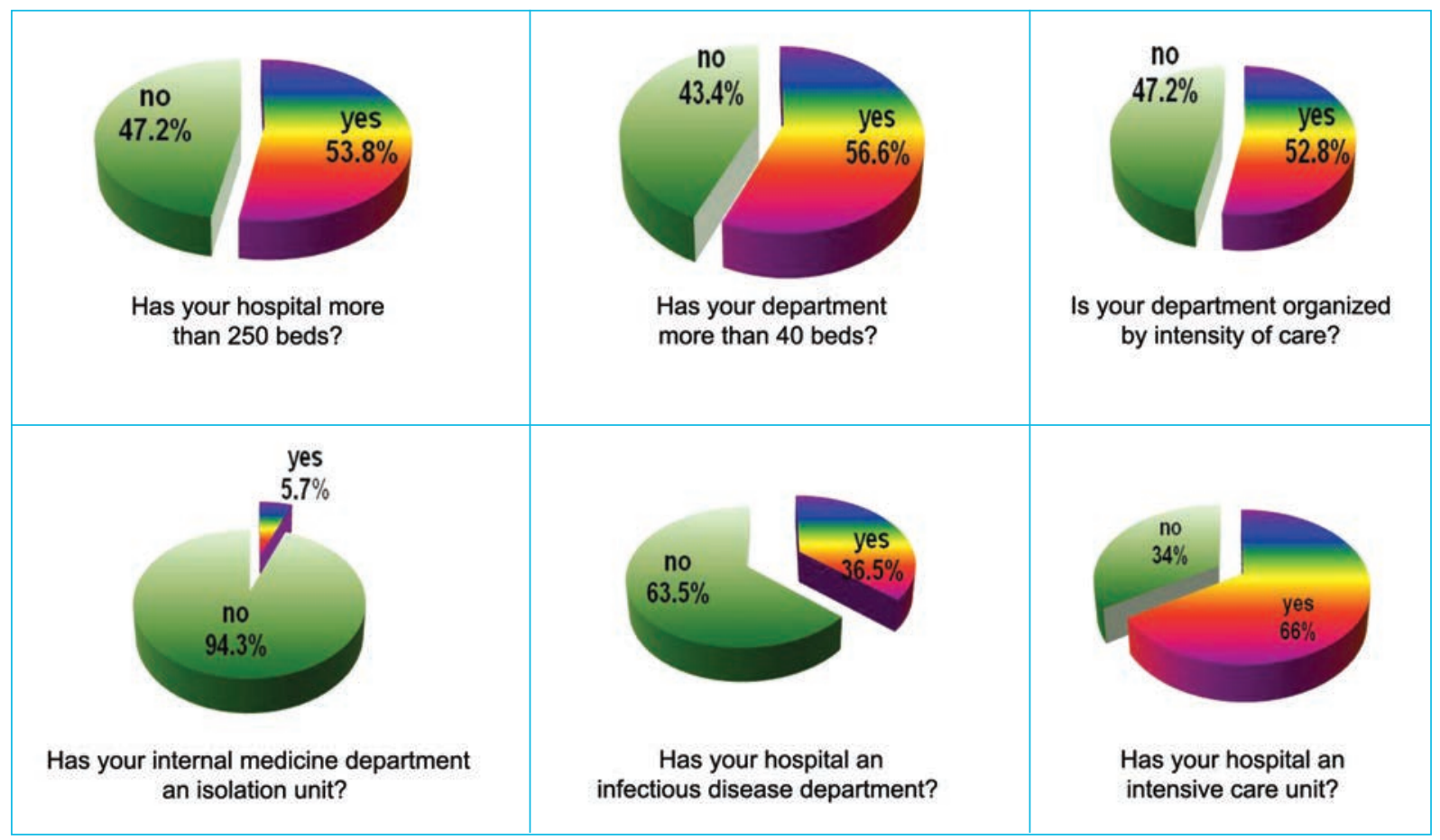

Figure 2. Structural features of the $\mathbf{5 3}$ departments taking part in the study. 
correct interpretation of the antibiogram when available, in order to reformulate or adequately reduce the therapy. Along this process, the collaboration with the clinical microbiologist is mandatory, who should provide in addition to the test results quickly, also the MIC sensitivity and a comment on the reference breakpoints developed by the European Committee on Antimicrobial Susceptibility Testing (EUCAST) and the Clinical and Laboratory Standard Institute (CLSI).

Our study is only a survey on the practices reported by the heads of the internal medicine departments in the Emilia Romagna region on the empirical use of antibiotics and is not intended to be in any way a regional registry on the use of antibiotics. It shows that in most cases the specialist who is more widely present throughout the region and in most cases is the first to approach the problem of severe infections is the internist who has a good (though certainly still improvable) awareness of this role. It cannot be denied that in this field it is important to involve more internists who usually practice other disciplines, since they are often called on to provide advice in strategic areas, such as ER and surgical wards, in particular where expertise on infectious diseases is not readily available for logistical reasons. As is well known, it is in the first hours (3-4) that the most appropriate decisions need to be made, since they can reasonably save the patient's life (filling volume, cardio-respiratory support, transfer to ICU, beginning of antibiotic therapy). This survey has then a full validity and highlights that also in a good clinical-organizational context there might be some inadequate behaviors that may require specific actions for improvement. One of the pivotal steps in the treatment of acute infections is the appropriateness of prescription which means, in other words, administering the right drug to the right patient. In the empirical therapy, this is just as important as the knowledge of the local epidemiology of infectious agents that can enable the healthcare providers to identify both the organisms most frequently involved in a given pathology and the spectrum of resistance.

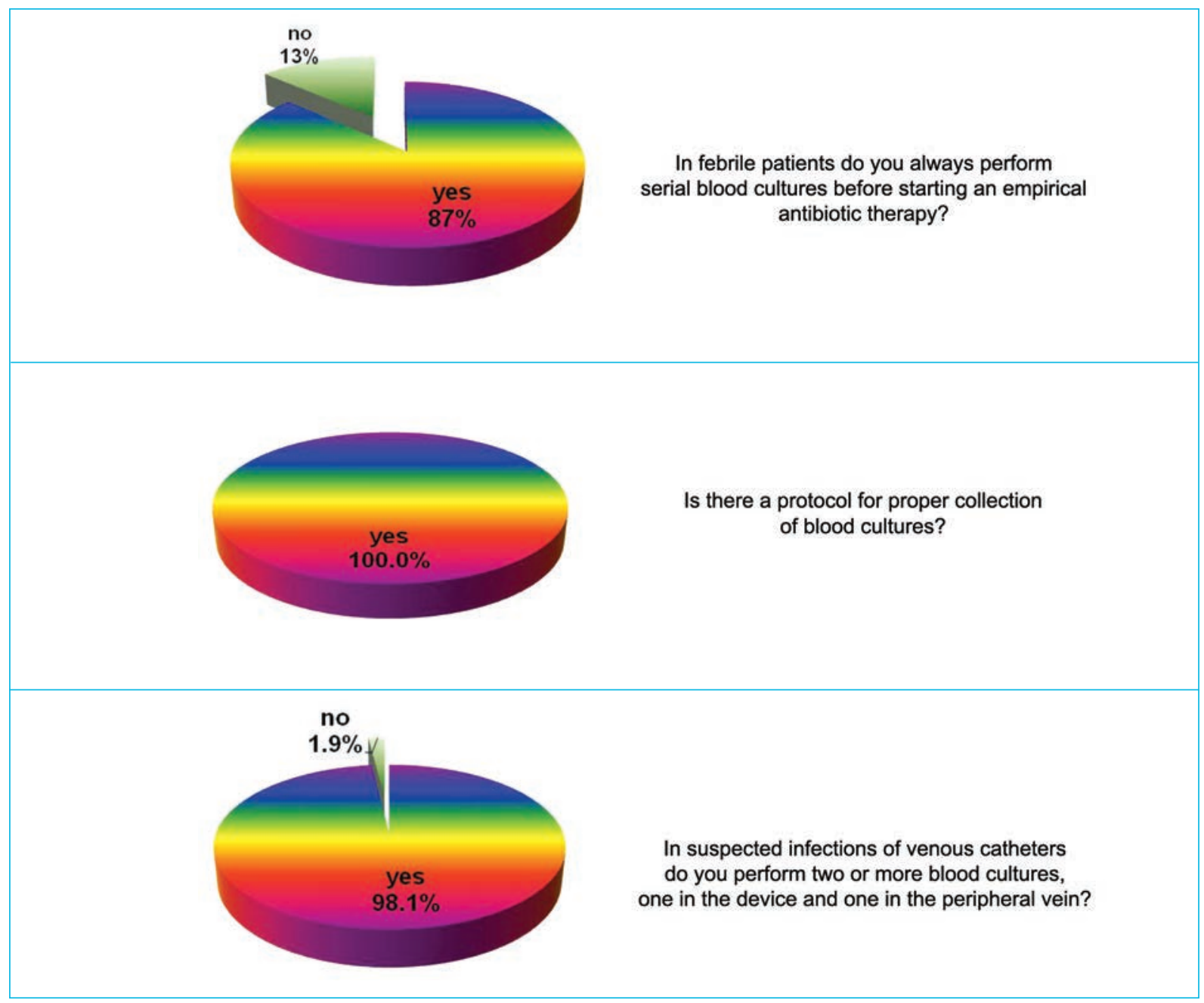

Figure 3. Management of blood cultures in the internal medicine departments. 
Once again the dialogue between the clinician and the microbiologist becomes critical in order to use correctly the most effective and appropriate molecule to achieve the best clinical outcome by treating successfully the disease and saving patients' lives and indirectly reduce hospital admission rates and healthcare costs.

This study shows that in the area of internal medicine and especially in small and peripheral hospitals, the use of the latest generation drugs (linezolid, tigecycline, daptomycin) to treat severe infections caused by Gram-positive bacteria is rather infrequent. This is sometimes the result of cost reduction efforts and constraints in the delivery of drugs by the pharmaceutical services. Likewise it reflects a sort of reluctance on the part of the internist to use expensive drugs, also in an attempt to avoid early resistance phenomena. However, in properly selected cases, this attitude can sometimes cause dangerous delays that might undermine the potential efficacy of the therapy.

An apparent contradiction is a generally wide, and not always appropriate, use of other molecules that, although not easy to use, have become part of the therapeutic armamentarium of the internist a long time ago. This refers to an improper utilization of glycopeptides (vancomycin and teicoplanin) in about $52 \%$ of the cases, as first choice treatment for MSSA infections. However, other molecules ( $\beta$-lactam antibiotics in the first place) are preferable, whereas the abovementioned drugs should not definitely be the first choice treatment in these infections, can also have severe side effects and must be used correctly (known or suspected MRSA infections), taking into account their pharmacokinetic and pharmacodynamic properties that require protracted doses in order to maintain an adequate concentration over time.

A distinguishing feature of this study is the analysis of the reporting tools available to the internists to monitor the use of drugs and the appropriateness of prescriptions depending on the local epidemiological situation. If, on the one hand, reports are significantly more present in large hospitals than in small ones, on the other in small hospitals information is more complete, because it does not only include administrative/economic data, but also organizational and clinical data and is frequently managed with modern computerized systems which replace printed documents.

In conclusion, this survey clearly shows that, both in large and small hospitals in the Emilia Romagna region, there is a need for proper stewardship of antibiotics, so that within the next few years it will be possible to use more properly and therefore wisely the antibiotic molecules available against both Gram positive and Gram negative infections. In particular, in smaller peripheral and local hospitals, the internist can be in charge of this process, ensures a continuous presence and acts on two distinct but closely complementary fronts. In fact, firstly, he/she is responsible for creating positive synergies with the infectious disease specialist in charge in order to build a common and adequate behavior, and secondly he/she promotes formal audit sessions on the use of antibiotics in the local community with the involvement of other professionals (health department, laboratory, pharmacy). In order to facilitate the development of such formal audit session and standard behaviors in all internal medicine departments in the region, a specific checklist aimed at constantly monitoring the behaviors of the clinical staff will be proposed in another dedicated publication. The adoption of these self-assessment models (internal audits) will reveal the consistency of therapeutic choices and highlight any discrepancies (complications, prolonged hospital stay duration, hospital readmission rates, etc.).

Table 1. Summary of the statistically significant variables.

\begin{tabular}{|c|c|}
\hline Is internal medicine advice sought by the emergency department for patients with acute infection? & $\begin{array}{c}\mathrm{P}<0.05 \\
\text { Small hospitals }\end{array}$ \\
\hline Is internal medicine advice sought by the surgical department for patients with acute infection? & $\begin{array}{c}\mathrm{P}<0.05 \\
\text { Small hospitals }\end{array}$ \\
\hline New drugs are used for MRSA bacterial infections with MIC Gram $+>$ or $=1$ vs glycopeptides linezolid & $\begin{array}{c}\mathrm{P}<0.05 \\
\text { Large hospitals }\end{array}$ \\
\hline New drugs are used for MRSA bacterial infections with MIC Gram $+>$ or $=1$ vs glycopeptides daptomycin & $\begin{array}{c}\mathrm{P}<0.05 \\
\text { Large hospitals }\end{array}$ \\
\hline Periodic reports on the antibiotic therapy are available: antibiotic class, average daily dose, mean duration of therapy & $\begin{array}{c}\mathrm{P}<0.05 \\
\text { Large hospitals }\end{array}$ \\
\hline Is data collection done with specific software programs? & $\begin{array}{c}\mathrm{P}<0.05 \\
\text { Small hospitals }\end{array}$ \\
\hline Do you test/use systems for measuring prescription results, resource consumption, effectiveness? & $\begin{array}{c}\mathrm{P}<0.05 \\
\text { Small hospitals }\end{array}$ \\
\hline
\end{tabular}

MRSA, methicillin-resistant staphylococcus aureus infections; MIC, mimimum inhibitory concentration. 


\section{References}

1. American Thoracic Society, Infectious Diseases Society of America. Guidelines for the management of adults with hospital-acquired, ventilator-associated, and healthcare-associated pneumonia. Am J Respir Crit Care Med 2005;171:388-416.

2. Shorr AF, Micek ST, Welch EC, et al. Inappropriate antibiotic therapy in gram-negative sepsis increases hospital length of stay. Crit Care Med 2011;39:46-51.

3. Lueangarun S, Leelarasamee A. Impact of inappropriate empiric antimicrobial therapy on mortality of septic patients with bacteremia: a retrospective study. Interdiscip Perspect Infect Dis 2012;2012:765205.

4. McKenzie C. Antibiotic dosing in critical illness. J Antimicrob Chemother 2011;66:ii25-31.

5. Ganguly NK, Arora NK, Chandy SG, et al. Rationalizing antibiotic use to limit antibiotic resistance in India. Indian J Med Res 2011;134:281-94.
6. McNulty CA, Kane A, Foy CJ, et al. Primary care workshops can reduce and rationalize antibiotic prescribing. J Antimicr Chemother 2000;46:493-9.

7. Kades E. Preserving a precious resource: rationalizing the use of antibiotics. Northwestern University Law Review; January 1, 2005. Available from: http://www.highbeam.com/doc/1P3-834011701.html

8. Dellinger RP, Levy MM, Rhodes A, et al. Surviving sepsis campaign: international guidelines for management of severe sepsis and septic shock: 2012. Crit Care Med 2013;41:580-637.

9. Rivers E, Nguyen B, Havstad S, et al. Early goal-directed therapy in the treatment of severe sepsis and septic shock. N Engl J Med 2001;345:1368-77.

10. Leibovici L, Shraga I, Drucker M, et al. The benefit of appropriate empirical antibiotic treatment in patients with bloodstream infection. J Intern Med 1998;244: 379-86.

\section{APPENDIX \\ Centers participating in the study}

Giuseppe Civardi Fiorenzuola d'Arda (PC)

Marco Grandi Sassuolo (Modena)

Giuseppe Chesi Scandiano (RE)

Giovanni Della Casa Vignola (MO)

Domenico Panuccio Bologna (BO)

Giorgio Cioni Pavullo nel Frignano (MO)

Casimiro Tramaloni Castel San Giovanni (PC)

Massimo Gallerani Ferrara (FE)

Giovani Fornaciari Reggio Emilia (RE)

Giorgio Ioli Santarcangelo di Romagna (RN)

Giuseppe Francesco Stefanini Faenza (RA)

Sergio Maccari Castelnovo nè Monti (RE)

Roberto Nardi Bologna (BO)

Antonino Praticò Bagno di Romagna (FC)

Roberto Quintavalla Parma (PR)

Maurizio Ongari Porretta Terme (BO)

Alberto Caiazza Borgo val di Taro (PR)

Maurizio Nizzoli Forli ( $F C$ )

Andrea Grossi Riccione (RN)

Matteo Giorgi Pierfranceschi Fiorenzuola d'Arda (PC)

Giampiero Pasini Cesena (FC)

Marcello Pradelli Baggiovara (MO)

Vittorio Durante Cattolica (RN)

Loris Borghi Parma (PR)

Paolo Gruppillo Argenta (FE)

Vincenzo Arienti Bologna (BO)

Giovanni Pedretti Fidenza (PR)
Valeria Manicardi Montecchio Emilia (RE)

Giovanni Iosa Cesenatico (FC)

Ido Iori Reggio Emilia (RE)

Paolo Tosi Mirandola (MO)

Giorgio Ballardini Rimini (RN)

Roberto Manfredini Ferrara (FE)

Carlo Cagnoni Bobbio (PC)

Alberto Bagnulo Correggio (RE)

Corrado Brignola Imola (BO)

Carlo Di Donato Carpi (MO)

Andrea Cuppini Budrio (BO)

Luigi Bolondi Bologna (BO)

Costanza Farabegoli Imola (BO)

Gianpaolo Bragagni San Giovanni in Persiceto (BO)

Sergio Orlando Piacenza (PC)

Giuseppe Re Lugo di Romagna (RA)

Mauro Silingardi Guastalla (RE)

Maurizio Ventrucci Bentivoglio (BO)

Marco Zoli Bologna (BO)

Gianfranco Cervellin Parma (PR)

Davide Imberti Piacenza (PC)

Enrico Fiaccadori Parma (PR)

Gianni Rastelli Fidenza (PR)

Maria Luisa Grata Ferrara (FE)

Giovanni Pinelli Baggiovara (MO)

Loris Montanari Ravenna (RA)

\section{Steering committee FADOI Emilia Romagna (Italy)}

Cioni Giorgio (President), Accogli Esterita, Ballardini Giorgio, Bragagni Gianpaolo, Chesi Giuseppe, Civardi Giuseppe, Gilioli Fabio, Giorgi Pierfranceschi Matteo, Giuri Pasquale Gianluca, Grandi Marco, Iosa Giovanni, Meschi Michele, Montanari Loris, Nardi Roberto, Nizzoli Maurizio, Ongari Maurizio, Panuccio Domenico, Pedretti Giovanni, Silingardi Mauro, Tirotta Daniela, Urbinati Sofia. 